\title{
Criminologie
}

\section{Mesure des symptômes dissociatifs chez des individus " borderlines " coupables de l'homicide de leur conjointe}

\section{Manon Blackburn et Gilles Côté}

Volume 34, numéro 2, automne 2001

Comportements suicidaires et délinquance

URI : https://id.erudit.org/iderudit/027508ar

DOI : https://doi.org/10.7202/027508ar

Aller au sommaire du numéro

Éditeur(s)

Les Presses de l'Université de Montréal

ISSN

0316-0041 (imprimé)

1492-1367 (numérique)

Découvrir la revue

Citer cet article

Blackburn, M. \& Côté, G. (2001). Mesure des symptômes dissociatifs chez des individus « borderlines » coupables de l'homicide de leur conjointe.

Criminologie, 34(2), 123-143. https://doi.org/10.7202/027508ar
Résumé de l'article

La violence conjugale est un phénomène fréquent. Statistiques Canada (1994) a évalué que trois femmes sur dix au Canada ont été victimes au moins une fois d'un acte de violence physique ou sexuelle de la part d'un conjoint. Au Canada, les homicides conjugaux représentent près de $12 \%$ de l'ensemble des homicides (Wilson et ai, 1995). L'homicide conjugal est généralement précédé par des incidents violents dans le couple. Les différentes formes de violence conjugale ne peuvent être graduées le long d'un con- tinuum de sévérité ; les typologies d'hommes violents le démontrent. L'objectif de cette recherche est d'identifier des facteurs associés à l'homicide dans un contexte de violence conjugale. L'hypothèse centrale porte sur la dissociation observée chez l'individu homi- cide suite à la perte de l'objet. L'étude comporte deux groupes de participants « borderlines » : 14 hommes coupables d'homicide conjugal, recrutés à l'intérieur de pénitenciers, et 14 hommes violents physiquement avec leur conjointe, recrutés dans des centres pour hommes violents. Les résultats au Hand Test (Wagner, 1983) et à l'échelle des expériences dissociatives (Bernstein et Putnam, 1986) sont comparés. 


\title{
Mesure des symptômes dissociatifs chez des individus "borderlines " coupables de l'homicide de leur conjointe
}

\author{
Manon Blackburn \\ Université du Québec à Trois-Rivières \\ Gilles Côté \\ Directeur \\ Centre de recherche de l'Institut Philippe Pinel de Montréal \\ Professeur \\ Departement de psychologie \\ Université du Québec à Trois-Rivières \\ gilles_cote@uqtr.uquebec.ca
}

RÉSumÉ - La violence conjugale est un phénomène fréquent. Statistiques Canada (1994) a évalué que trois femmes sur dix au Canada ont été victimes au moins une fois d'un acte de violence physique ou sexuelle de la part d'un conjoint. Au Canada, les homicides conjugaux représentent près de $12 \%$ de l'ensemble des homicides (Wilson et al., 1995). L'homicide conjugal est généralement précédé par des incidents violents dans le couple. Les différentes formes de violence conjugale ne peuvent ètre graduées le long d'un continuum de sévérité ; les typologies d'hommes violents le démontrent. L'objectif de cette recherche est d'identifier des facteurs associés à l'homicide dans un contexte de violence conjugale. L'hypothèse centrale porte sur la dissociation observée chez l'individu homicide suite à la perte de l'objet. L'étude comporte deux groupes de participants "borderlines " : 14 hommes coupables d'homicide conjugal, recrutés à l'intérieur de pénitenciers, et 14 hommes violents physiquement avec leur conjointe, recrutés dans des centres pour hommes violents. Les résultats au Hand Test (Wagner, 1983) et à l'échelle des expériences dissociatives (Bernstein et Putnam, 1986) sont comparés.

abstract - Conjugal violence is a frequent phenomenon. Statistics Canada (1994) estimated that three out of ten women in Canada have been victimized physically or sexually at least once by a spouse. In Canada, spousal homicides represent nearly $12 \%$ 
of all homicides (Wilson et al., 1995). Spousal homicide is generally preceded by acts of violence between spouses. The various forms of conjugal violence cannot be graded along a continuum of severity, as evidenced by the typologies for violent men. The purpose of this study was to identify factors associated with homicide within the context of conjugal violence. The central hypothesis concerned the dissociation observed in homicide patients following loss of object. The study involved two groups of borderline subjects : 14 men convicted of spousal homicide (recruited in penitentiaries) and 14 men physically violent with their spouses (recruited in centres for violent men). Results on the Hand Test (Wagner, 1983) and on the Dissociative Experiences Scale (Bernstein et Putnam, 1986) were compared.

\section{Contexte théorique}

La violence conjugale est un phénomène fréquent. Statistiques Canada (1994) a évalué que trois femmes sur dix au Canada - mariées ou l'ayant été, ou vivant en union libre - ont été victimes au moins une fois d'un acte de violence physique ou sexuelle, proscrit par la loi, de la part d'un conjoint ou d'un ex-conjoint. Cette violence s'exprime parfois par l'homicide. Dans une étude classique faite à Philadelphie, Wolfgang (1958) observe que $24.7 \%$ des homicides se produisent dans le cadre de relations familiales; de ce nombre, la moitié sont commis contre un conjoint ou une conjointe. Dans une étude plus récente, Wilson $e t$ al. (1995) observent que les homicides conjugaux au Canada représentent près de $12 \%$ de l'ensemble des homicides.

Les homicides commis au sein de la famille sont particuliers. Ceuxci résultent de conflits engendrés dans une relation intime; ils sont le fait d'individus qui, généralement, ont la capacité de bien s'adapter à la société, d'une part (Cormier et al., 1971 ; Daniel et Holcomb, 1985 ; Kalichman, 1988 ; Crawford et Gartner, 1992), et qui ne présentent pas de maladie mentale suffisamment grave qui les rendrait inaptes à subir leur procès, d'autre part (Cormier et al., 1971 ; Lester, 1975 ; Chimbos, 1978 ; Duncan et Duncan, 1978 ; Barnard et al., 1982 ; Côté, 1991 ; Saunders et Browne, 1991). Ces homicides sont généralement faits de façon impulsive sans qu'il y ait eu préméditation de la part de l'individu homicide (Lester, 1975 ; Showalter et al., 1980 ; Kratcoski, 1987).

Les femmes sont, le plus fréquemment, les victimes des homicides conjugaux (Horoszowski, 1973 ; Daniel \& Holcomb, 1985 ; Straus, 1986 ; Kratcoski, 1987 ; Côté, 1991 ; Boisvert et Cusson, 1999). Par ailleurs, plusieurs auteurs constatent que, généralement, la femme qui tue son conjoint commet un acte d'autodéfense après des années d'abus 
sévères (Wolfgang, 1958 ; Barnard et al., 1982 ; Straus, 1986 ; Goetting, 1989b ; Saunders et Browne, 1991 ; Cooper et Eaves, 1996 ; Okeefe, 1997).

Les motivations qui poussent les hommes à tuer leur conjointe semblent différentes d'un individu à l'autre, mais elles se situent généralement dans un contexte où la violence conjugale règne. La plupart du temps, l'homicide est précédé d'une série d'incidents agressifs et de disputes dans le couple (Cormier, 1962 ; Boudouris, 1971 ; Chimbos, 1978 ; Showalter et al., 1980 ; Barnard et al., 1982 ; Straus, 1986 ; Kratcoski, 1987 ; Daly et Wilson, 1988 ; Goetting, 1989a ; Saunders et Browne, 1991 ; Wilson et Daly, 1993 ; Campbell, 1995 ; Ewing, 1997 ; Walker et Meloy, 1998).

Il est difficile de prévoir l'homicide conjugal, pour deux motifs principaux : il y a peu d'hommes violents qui vont tuer leur conjointe, et les variables identifiées comme jouant un rôle dans la violence mortelle et dans la violence non mortelle sont souvent les mêmes. Chez les deux groupes d'hommes, définis par le type de violence, le statut économique est généralement bas et le niveau d'éducation faible (Horoszowski, 1973 ; Chimbos, 1978 ; Coleman et al., 1980 ; Gelles, 1980 ; Showalter et al., 1980 ; Hamberger et Hasting, 1988 ; Goetting, 1989a ; Crawford et Gartner, 1992 ; Campbell et Landenburger, 1995 ; Saunders, 1995 ; Wilson et al., 1995).

L'âge moyen du conjoint homicide au moment de l'acte est de 35 ans (Chimbos, 1978 ; Showalter et al., 1980 ; Rosenberg et Mercy, 1986 ; Goetting, 1989a ; Crawford et Gartner, 1992). Il semble légèrement moins élevé en ce qui concerne la violence non mortelle. Statistiques Canada (1994) rapporte des taux plus élevés de violence conjugale chez les jeunes de 18 à 24 ans. Edleson et al. (1985) situent, quant à eux, l'âge moyen des hommes violents entre 20 et 30 ans.

Avoir été victime ou témoin de violence durant son enfance, à l'intérieur de sa famille d'origine, est un facteur important associé au fait d'être violent avec sa conjointe à l'âge adulte; ce facteur est retrouvé autant chez les individus violents non homicides (Tolman et Bennett, 1990) que chez les individus homicides (Chimbos, 1978). D'autres études sur l'enfance des individus violents montrent que la majorité des hommes qui ont tué leur conjointe ont connu une enfance marquée par le rejet de la part de leurs parents (Showalter et al., 1980 ; Daniel et Holcomb, 1985). 
Plus de la moitié des hommes violents avaient consommé des substances psychoactives lors des incidents de violence conjugale de tout ordre $(61 \%)$ et plusieurs connaissaient des problèmes d'abus chroniques ou de dépendance par rapport à l'alcool ou à la drogue (53\%) (Tolman et Bennett, 1990). Dans les cas des homicides conjugaux, la consommation d'alcool avant le crime varie de $32 \%$ à $75 \%$, selon les échantillons (Chimbos, 1978 ; Daniel et Holcomb, 1985 ; Crawford et Gartner, 1992 ; Gondolf et Shestakov, 1997) ; les habitudes de consommation à des moments autres que lors du crime ne sont pas précisées.

Plusieurs études signalent que les hommes qui tuent leur conjointe le font souvent suite à une séparation, une menace de séparation ou un incident ayant provoqué une réaction de jalousie. En somme, l'homicide est, la plupart du temps, précédé d'un sentiment d'abandon ou de rejet ressenti par le sujet homicide (Cormier, 1962 ; Showalter et al., 1980 ; Barnard et al., 1982 ; Rosenbaun, 1990 ; Côté, 1991 ; Crawford et Gartner, 1992 ; Wilson et Daly, 1993 ; Polk, 1994 ; Cooper et Eaves, 1996 ; Gondolf et Shestakov, 1997 ; Walker et Meloy, 1998 ; Boisvert et Cusson, 1999). Wilson et al. (1995) soutiennent que la violence conjugale, autant mortelle que non mortelle, est reliée à des attitudes de jalousie et de possession chez l'homme et à des désertions réelles ou imaginaires de la femme. Dutton et Golant (1996), de même que Dutton (1998), reconnaissent que les incidents violents sont effectivement précédés d'une rage ressentie face à des menaces d'abandon et de rejet ; ils précisent que, dans des situations extrêmes où il y a un processus de rumination, la violence peut se rendre jusqu'à l'homicide.

Bien que les recherches sur l'homicide conjugal soient surtout axées sur des aspects sociodémographiques, certains auteurs constatent une fréquence élevée de troubles de la personnalité dans leur échantillon, plus particulièrement des troubles de la personnalité de types passifdépendant, " borderline " et antisocial (Showalter et al., 1980 ; Rosenbaum, 1990 ; Yarvis, 1990). Les troubles de la personnalité sont aussi fréquemment retrouvés dans les échantillons d'hommes violents ; on les rencontre tout particulièrement chez les hommes qui souffrent de problèmes liés à l'abus de substances psychoactives (Hotaling et Sugarman, 1986 ; Hamberger et Hasting, 1988 ; Tolman et Benett, 1990 ; Saunders, 1995). Hamberger et Hasting (1986), à partir d'un échantillon de 99 individus, observent que près de $88 \%$ des hommes violents souffrent d'un trouble de la personnalité. Avec ces résultats, ils arrivent à classifier ces hommes en trois sous-groupes sur la base de leur 
personnalité, soit les sous-groupes schizoïde/ «borderline », narcissique/antisocial et passif-dépendant/ compulsif.

Certaines études mettent aussi en évidence trois types distincts d'hommes violents (Saunders, 1992 ; Holtzworth-Munroe et Stuart, 1994 ; Dutton, 1998 ; Walker et Meloy, 1998). Ces études abordent une classification en fonction de la portée de la violence (violence domestique ou violence à l'extérieur de la maison), de la sévérité de la violence et des profils psychologiques, lesquels se situent généralement dans le champ des troubles de la personnalité. Dutton (1998) donne un aperçu intéressant de ces trois types en les dispersant sur deux axes, soit l'axe allant de la violence instrumentale à la violence impulsive et l'axe qui va du manque de contrôle au "surcontrôle". Il trouve trois types d'hommes violents : les psychopathes qui présentent des caractéristiques antisociales, les "surcontrôlés ", caractérisés par une personnalité évitante, passive-agressive ou dépendante, et les " cogneurs cycliques", lesquels ont une personnalité "borderline ». C'est sur ce dernier type que Dutton base l'ensemble de ses recherches. L'homicide pourrait, dans cette perspective, être un acte accompli par un type particulier d'hommes violents.

Plusieurs de ces résultats amènent à penser que l'homicide est l'aspect le plus visible de la violence conjugale. Les théories qui tentent d'expliquer l'homicide sont en grande partie les mêmes que celles utilisées pour expliquer la violence familiale sous toutes ses formes. Il est question de cas extrêmes ou de situations extrêmes pour expliquer l'homicide. Toutefois, les auteurs n'incluent pas de groupes de comparaison, composés d'individus homicides, dans leurs études, ce qui permettrait de départager ce qui appartient à l'homicide conjugal, d'une part, et ce qui appartient à la violence conjugale non mortelle, d'autre part. Wilson et al. (1995) comparent la violence conjugale mortelle et non mortelle, mais ils le font surtout à partir de variables démographiques telles que l'âge et les statuts conjugal et économique. Dans les faits, ce n'est qu'une minorité d'hommes violents envers leur conjointe qui vont commettre l'homicide. Saunders et Browne (1991) notent que, sur 108000 hommes et femmes qui ont utilisé des fusils ou des couteaux contre leur partenaire au cours de l'année 1985 , moins de $2 \%$ des cas, seulement, ont eu pour résultat la mort de la victime. Il n'est donc pas surprenant que l'homicide soit étudié sans être distingué des autres formes de violence.

Cette façon d'étudier l'homicide laisse supposer que les gestes de violence se répartissent sur un continuum de sévérité. Même si les 
recherches sur la violence conjugale montrent une progression de la violence verbale vers des formes de violence physique plus graves (Sugarman et al., 1996), l'hypothèse d'un continuum ne peut expliquer des formes spécifiques de violence comme l'homicide. D'une part, cette hypothèse intègre mal le fait que les hommes violents ne constituent pas un groupe homogène. D'autre part, de façon générale, les individus homicides sont distincts des individus non homicides; ils présentent plus souvent un trouble mental grave (Côté et Hodgins, 1992). Steinmetz (1978), Block (1987) et Côté et al. (1999) estiment tous que les diverses formes de violence ne peuvent être différenciées sur la seule base de leur sévérité ; ils critiquent la conception d'un continuum de la violence et ils proposent plutôt de parler de phénomènes ou de dynamiques distincts pour différencier les formes de violence.

Duncan et Duncan (1978) décrivent l'acte homicide dans le couple comme une tentative pour résoudre un conflit intrapsychique dévastateur provoqué par l'incapacité de se retirer d'une relation qui est devenue intolérable. Barnard et al. (1982) soulignent les difficultés éprouvées par ces hommes face au rejet ; ceci affecterait directement leur identité ou l'image de leur rôle sexuel qui repose sur le désir de dominer. Selon ces auteurs, cet aspect différencie les hommes et les femmes dans le cadre de l'homicide conjugal. Chimbos (1978) pense aussi que l'acte homicide permet de restaurer un moi faible chez des hommes qui s'estiment peu et qui présentent une capacité verbale limitée.

Dans leur échantillon d'hommes ayant commis des actes violents graves ou ayant tué leur conjointe, Showalter et al. (1980) notent que, dans chaque cas, les individus ont identifié la relation avec leur femme comme étant la première ou la seule relation intime qu'ils ont été capables de développer. Cormier et al. (1962) identifient un lien de dépendance entre les deux partenaires; la gratification de l'homme dépend exclusivement de sa relation avec sa conjointe. Malmquist (1980) parle de difficulté de séparation/individualisation. Selon lui, l'homicide conjugal se produit dans un état d'indifférenciation entre le soi et le non-soi qui est maintenu par des traits masochistes retrouvés chez l'offenseur.

Certains auteurs expliquent le passage à l'acte homicide contre la conjointe par la dissociation ou le clivage de l'objet lors du crime. Cormier et al. (1971) font la distinction entre les meurtriers psychotiques et les meurtriers " normaux » ou non psychotiques. Ils formulent l'hypothèse que les homicides commis à l'intérieur d'une relation intime 
sont exécutés par des individus non psychotiques qui régressent, pendant une courte période, dans un état dissociatif où prédominent des mécanismes psychotiques. Ce passage vers un état dissociatif est décrit comme une régression vers des mécanismes psychotiques suite au désespoir et à la rage ressentis devant l'éventualité de perdre l'objet. Dans un contexte de rejet, le bon objet se transforme en mauvais objet qui est tué de façon impulsive. Blinder $(1984 ; 1985 a ; 1985 b)$ parle des «tueurs dissociatifs" : des individus particulièrement fragiles, qui arrivent ordinairement à s'adapter, mais qui sont incapables de tolérer les fluctuations de la vie conjugale. Un état de dissociation s'installe où cumule leur colère. L'auteur décrit l'état de dissociation comme un mécanisme de défense, un clivage essentiellement inconscient pour se défendre contre la détresse émotionnelle et atténuer les situations menaçantes. Selon lui, la dissociation meurtrière et aiguë se produit lorsque la personne découvre que sa survie dépend de la réconciliation de l'irréconciliable dans son couple.

Dans ces études, l'hypothèse de la dissociation n'est toutefois pas mesurée de façon formelle. Dutton, Ginkel et Landolt (1996), de même que Dutton, Starzomski et Ryan (1996), examinent certains symptômes de dissociation dans la violence non mortelle. Ils observent chez des hommes violents des symptômes reliés au choc post-traumatique (dépression, anxiété, trouble du sommeil et symptômes rattachés à la dissociation, tels que dédoublements, retours en arrière, vertiges et expériences de sortie du corps). Les hommes violents ont un profil comparable aux hommes qui, dans d'autres études, ont rencontré les critères du syndrome post-traumatique. Dans ces mêmes études, Dutton et ses collègues trouvent des associations entre la jalousie, l'attachement craintif, la personnalité « borderline " et les états de stress post-traumatique. À partir de ces données, ils font l'hypothèse que les traumatismes vécus dans l'enfance, alliés à un attachement insécure, seraient à l'origine d'une personnalité qui craint l'abandon et qui réagit de façon pathologique à la séparation : la personnalité «borderline ». Selon eux, la violence est moins attribuable à une cause physiologique, génétique ou sociale, qu'à un fondement psychologique prenant sa source dans le développement précoce de la personnalité. Dutton (1998) soutient l'hypothèse que les hommes qui frappent leur conjointe ne forment plus une, mais deux personnes au moment d'entrer dans des phases violentes.

Le phénomène de l'homicide conjugal pourrait donc être défini comme une régression vers un état de dissociation chez des individus 
ayant une fragilité intrapsychique face à la menace de perte de l'objet. Cet état de fait pourrait se retrouver chez des individus «borderlines» se situant sur l'échelon inférieur de l'organisation « borderline » dans le cadre de la compréhension des états " borderlines", tels que décrits par Kernberg (1976). Bergeret (1995) parle de prépsychotiques pour identifier ces sujets ; il les décrit comme des sujets ayant déjà une relation objectale de mode psychotisant, sans que les dénis soient assez conséquents pour introduire des formations imaginaires délirantes. Cette description définit bien l'aménagement psychique des individus " borderlines", tel que mentionné par Gunderson (1984). Cet auteur décrit trois niveaux de fonctionnement chez les personnalités «borderlines». Lorsque de tels individus se sentent en contact avec un objet qui les supporte, le sens de la réalité est intact (niveau 1). Quand l'objet se fait menaçant, par des frustrations ou la possibilité d'une perte, le sens de la réalité devient fragile, mais demeure toujours intact (niveau 2). Lorsque l'objet devient absent, ou bien le sens de la réalité devient fragile, ou bien des phénomènes de dissociation, qualifiés parfois de phénomènes de dépersonnalisation, se produisent ; ces phénomènes correspondent à des tentatives intrapsychiques de restaurer l'objet perdu (niveau 3).

En regard de l'ensemble de ces informations, l'objectif de l'étude est d'identifier des facteurs associés à l'homicide, dans un contexte de violence conjugale. L'hypothèse centrale est que les hommes "bordetlines" coupables de l'homicide de leur conjointe présentent significativement plus d'indices dissociatifs que les hommes "borderlines" qui sont violents physiquement envers leur conjointe, sans avoir commis un homicide.

\section{Méthode}

\section{Participants}

Le groupe homicide (groupe $\mathrm{H}$ ) et le groupe non homicide (groupe $\mathrm{NH}$ ) sont composés respectivement de 14 hommes francophones rencontrant les critères du trouble de la personnalité «borderline » et ne rencontrant pas les critères d'un trouble bipolaire ou psychotique. Les participants du groupe $\mathrm{H}$ ont été reconnus coupables de l'homicide de leur conjointe ; ils ont été choisis dans des établissements québécois du Service Correctionnel du Canada. Le taux de refus qui tient compte des désistements survenus pendant toute l'étude est de $26,3 \%$ pour ce 
groupe. Dix participants ne répondaient pas aux critères de sélection : six étaient anglophones, trois ne rencontraient pas les critères du trouble de la personnalité " borderline » et un participant avait tué une femme qu'il ne reconnaissait pas comme sa conjointe. Le groupe NH est constitué de participants ayant été violents physiquement avec leur conjointe ; leur recrutement a été fait dans des organismes québécois spécialisés dans le traitement des hommes violents : le centre le CAHO et le centre Accord Mauricie Inc. Le taux de refus est de $56,3 \%$ pour ce groupe ; seul un sujet ne répondait pas aux critères du trouble de la personnalité " borderline ». Les deux groupes ne présentent pas de différences significatives quant à l'âge $(t(26)=.02, n .5)$. L'âge moyen est de 34.4 ans pour le groupe $\mathrm{H}$ et de 34.4 ans pour le groupe $N H$. Les deux groupes sont aussi comparables quant au statut économique $\left(\chi_{x}{ }^{2}\right.$ $(1, N=28)=0, n . s$.) et au niveau de la scolarité $\left(\chi_{x}^{2}(1, N=28)=3.6, n . s\right)$. La majorité des participants se retrouvent dans la catégorie des emplois non spécialisés ou sont sans emploi (57\%). La scolarisation est faible, $64 \%$ des participants homicides n'ont pas terminé leur secondaire; pour le groupe $\mathrm{NH}$, ce taux est de $30 \%$. Le fait que la différence ne soit pas statistiquement significative $(p=.058)$ en ce qui concerne la scolarisation peut s'expliquer par une puissance statistique limitée.

\section{Instruments de mesure}

Questionnaire psychodémographique. Ce questionnaire comprend des questions psychodémographiques ainsi que des questions ouvertes sur le contexte de l'homicide et le contexte des relations conjugales.

The Structured Clinical Interview for DSM-IV (SCID I et II) (First et al., 1997 ; Barden). Cet instrument permet de diagnostiquer les individus "borderlines " et d'éliminer les individus ayant des symptômes psychotiques ou bipolaires. Les échelles suivantes sont utilisées: désordres psychotiques, désordres de l'humeur et troubles de la personnalité. L'accord interjuges est parfait pour ces trois échelles, dans le cadre de la présente étude $(k=1.00)$.

The Conflict Tactics Scale (CTS) (Straus, 1979 ; Straus et Gelles, 1990). Ce test est utilisé pour évaluer la violence vécue dans le couple l'année précédant l'homicide. Shumm et Bagarozzi (1989) rapportent que seulement cinq études sur 17 montrent un alpha plus petit que .80 pour ce test. La validation franco-québécoise du CTS (Questionnaire sur la résolution des conflits, $\mathrm{QRC}$ ) montre que l'instrument peut fidè- 
lement discriminer les adultes violents des adultes non violents (Laferrière et Bouchard, 1993).

Dissociative Experiences Scale (DES) (Bernstein et Putnam, 1986). Cet instrument est constitué de vingt-huit items cotés sur une échelle de 0 à 100 . Ce test repose sur une approche descriptive basée sur les expériences de dissociation qu'a pu vivre l'individu dans sa vie. Il permet de dépister des individus ayant d'importants symptômes dissociatifs. Plusieurs recherches montrent que le DES est un bon instrument; la consistance interne atteint une moyenne de .87 dans l'ensemble des recherches, la fidélité, mesurée par des tests-retests, atteignant une moyenne de 93 (Bernstein et Putnam, 1986 ; Carlson et Putnam, 1993 ; Dubester et Braun, 1995 ; Gleaves et al., 1995 ; Ross et al., 1995 ; Van Ijzendoorn et Schuengel, 1996). L'instrument existait en version française (Malarewitz, 1990), mais la traduction paraissait inadéquate. Une seconde traduction a été effectuée et distribuée à deux groupes : 138 participants recrutés selon la méthode dite «boule de neige » et 145 recrutés dans deux classes d'étudiants en psychologie à l'Université du Québec à Trois-Rivières. Les analyses statistiques démontrent une bonne consistance interne de la version traduite de l'instrument : un alpha standardisé de .88 pour l'ensemble des participants, de .89 pour le groupe 1 et de .93 pour le groupe 2 . Les coefficients de cohérence interne (Split-half) de Spearman Brown sont de 78 pour la partie 1 et de 86 pour la partie 2 . Ces coefficients ont été établis sur la base d'une dichotomie de l'item définie à partir de la médiane.

Hand Test (Wagner, 1983). Ce test projectif est utilisé pour vérifier les indices dissociatifs à l'aide des scores "Failure " (FAIL) et "Description » (DESC); il s'agit respectivement de réponses pour lesquelles le participant n'arrive pas à préciser un contenu ou dans lesquelles il tente un contrôle de la projection à travers un contenu peu engageant. Dans ce dernier cas, il se contente de décrire très sommairement quelques traits physiques de la main. Ces types de réponses démontrent soit une téaction défensive ou faible avec la réalité (DESC), soit des tendances dissociatives ou de perte de contact avec la réalité (FAIL) (Wagner, 1983). Le Hand Test permet de mettre en évidence des modes de fonctionnement inconscient d'ordre structurel. Pour les réponses FAIL et DESC, l'accord interjuges est bon $(k=.60)$. 


\section{Déroulement}

Tous les participants ont signé un formulaire de consentement éclairé les informant des modalités de la recherche. Une procédure visant à assurer un accord interjuges a été utilisée pour le SCID et le Hand Test. Pour ce faire, les neuf premiers participants ont été rencontrés en présence de deux évaluateurs. Ces derniers ont reçu une formation sur la passation des deux tests, ainsi qu'une supervision durant toute l'étude. Tous les protocoles de Hand Test ont été d'abord cotés séparément par les deux évaluateurs, puis révisés, un par un, en présence d'un troisième évaluateur expérimenté.

\section{Résultats}

La majorité des participants ne présente pas de symptômes dissociatifs. Au DES, 23 participants (82.1\%) obtiennent un score $<20$, ce qui reflète l'absence de symptômes dissociatifs. Au Hand Test, 24 participants $(85.7 \%)$ n'obtiennent pas de score DESC ou FAIL. Le test MannWhitney ne montre aucune différence significative entre les groupes sur le score au DES $(M-W(28)=96, n . s$.$) . Les analyses de khi-carré ne$ montrent pas de différence significative entre les deux groupes $\left(\chi^{2}(1, N=28)=1.17, n . s.\right)$ pour les symptômes dissociatifs au Hand Test (voir Tableau 1).

Par ailleurs, huit participants homicides (57\%), questionnés à propos du contexte de leur homicide, mentionnent des phénomènes qu'on peut rattacher à des symptômes dissociatifs, tels que la perte de mémoire ou des phénomènes de dépersonnalisation. De plus, le crime est commis la plupart du temps dans un contexte où l'homme "borderline " se sent

TABLEA U 1

Comparaison des participants sur la base des indices de dissociation

\begin{tabular}{|c|c|c|c|c|}
\hline \multirow[b]{2}{*}{ Indices de dissociation } & \multirow{2}{*}{$\begin{array}{c}\text { Non } \\
\text { homicide }\end{array}$} & \multirow[b]{2}{*}{ Homicide } & \multicolumn{2}{|c|}{ Test de signification } \\
\hline & & & Type & $p$ \\
\hline $\begin{array}{l}\text { DESC et FAIL au Hand Test } \\
\text { (absence) } \\
\text { (présence) }\end{array}$ & $\begin{array}{c}11 \\
3\end{array}$ & $\begin{array}{c}13 \\
1\end{array}$ & Khi-carré & $>.05$ \\
\hline Score au DES (médiane) & 8.75 & 7.68 & Man-Whitney & $>.05$ \\
\hline
\end{tabular}


rejeté ou abandonné ; $85.7 \%$ des homicides sont précédés d'une séparation, d'une menace de séparation, d'un adultère ou d'un incident ayant provoqué une réaction de jalousie. Il n'existe pas de différence statistiquement significative entre les deux groupes $\left(\chi^{2}(1, N=28)=1.47\right.$, n.s. $)$ quant au nombre de séparations vécues dans le passé et quant à la durée de la dernière relation conjugale $(M-W(28)=79, n . s$.$) (voir Tableau 2).$ Seules les relations conjugales où il $\mathrm{y}$ a eu cohabitation ont été retenues pour ces deux dernières analyses.

Les participants du groupe NH disent avoir consommé des substances psychoactives, lors de la plupart des incidents de violence physique avec leur conjointe, dans une proportion de $31 \%$. Pour le groupe $\mathrm{H}$, cette proportion est de $29 \%$. Il n'existe pas de différence significative entre les deux groupes quant à cette variable $\left(\chi^{2}(1, N=20)\right.$ $=.01, n . s$.). La consommation de substances psychoactives est présente au moment de l'homicide dans $50 \%$ des cas.

Une différence significative est trouvée entre les groupes en ce qui concerne la durée totale des consultations psychologiques $\left(\chi^{2}(1, N=25)=6.99, p<.01\right)$. Dans le groupe $\mathrm{H}$, neuf participants $(69 \%)$ ont consulté un ou des professionnels en relation d'aide pendant plus de 12 mois. Dans le groupe $\mathrm{NH}$, deux participants ( $17 \%$ ) avaient à leur actif des consultations de plus de 12 mois. Il faut noter que, pour le groupe $\mathrm{H}$, la grande majorité des consultations ont été faites suite à l'homicide, dans des programmes offerts par les services correctionnels.

TABLEAU 2

Comparaison des participants sur diverses variables associées

\begin{tabular}{|l|c|c|c|c|}
\hline & \multirow{2}{*}{$\begin{array}{c}\text { Non } \\
\text { homicide }\end{array}$} & Homicide & \multicolumn{2}{|c|}{ Test de signification } \\
\cline { 4 - 5 } & 11 & 8 & Khi-carré & $>.05$ \\
\hline $\begin{array}{l}\text { Séparations passées } \\
\text { (présence) } \\
\text { Durée de la dernière } \\
\text { relation en mois (médiane) }\end{array}$ & 42 & 84 & Man-Whitney & $>.05$ \\
$\begin{array}{l}\text { Consommation de } \\
\text { substances lors des } \\
\text { incidents violents (\%) }\end{array}$ & 31 & 29 & Khi-carré & $>.05$ \\
$\begin{array}{l}\text { Durée des consultations } \\
\text { psychologiques } \\
\text { (+ de } 12 \text { mois) (\%) }\end{array}$ & 17 & 69 & Khi-carré & $<.01$ \\
\hline
\end{tabular}


En moyenne, les participants des deux groupes ont répondu aux critères de deux troubles de la personnalité. Une série d'analyses de khicarré n'a pas permis de différencier les deux groupes quant à la prévalence de certains types de troubles de la personnalité. Le Tableau 3 illustre les diagnostics multiples de troubles de la personnalité retrouvés pour chaque groupe.

TABLEAU 3

Répartition des diagnostics de troubles de la personnalité

\begin{tabular}{|c|c|c|c|}
\hline Diagnostics sur l'axe II & $\begin{array}{c}\text { Non } \\
\text { homicide }\end{array}$ & Homicide & Total \\
\hline «Borderline » & 6 & 5 & 11 \\
\hline "Borderline" - obsessionnelle-compulsive & 3 & 1 & 4 \\
\hline “Borderline »-paranoïaque & & 1 & 1 \\
\hline "Borderline » - antisociale & 2 & 3 & 5 \\
\hline "Borderline »- évitante - antisociale & & 2 & 2 \\
\hline $\begin{array}{l}\text { "Borderline »- évitante - obsessionnelle- } \\
\text { compulsive }\end{array}$ & 1 & & 1 \\
\hline "Borderline »-paranoïaque - antisociale & 1 & & 1 \\
\hline « Borderline » - schizoïde - antisociale & & 1 & 1 \\
\hline $\begin{array}{l}\text { "Borderline » - évitante - obsessionnelle- } \\
\text { compulsive - antisociale }\end{array}$ & & 1 & 1 \\
\hline $\begin{array}{l}\text { "Borderline »-obsessionnelle-compulsive } \\
\text { - paranoïaque - narcissique - antisociale }\end{array}$ & 1 & & 1 \\
\hline Total & 14 & 14 & 28 \\
\hline
\end{tabular}

Il n'y a pas de différence statistiquement significative entre les groupes sur le score total au CTS $(t(26)=.92, n$.s.) et sur le score à la souséchelle violence psychologique $(t(26)=96, n$.s.). Les analyses non paramétriques ne montrent pas de différence significative entre les groupes à la sous-échelle violence physique sévère $(M-W(28)=86, n . s)$, mais montrent une différence significative entre les deux groupes à la sous-échelle violence physique mineure $(M-W(28)=39, p<.01)$ (voir Tableau 4). 
TABLEAU 4

Résultats au CTS

\begin{tabular}{|l|c|c|c|c|}
\hline \multirow{2}{*}{ Indices } & $\begin{array}{c}\text { Non } \\
\text { homicide }\end{array}$ & Homicide & Type & $p$ \\
\cline { 4 - 5 } & 16.64 & 14.21 & Test-t & $>.05$ \\
\hline $\begin{array}{l}\text { Échelle violence } \\
\text { psychologique (moyenne) }\end{array}$ & 2.5 & .79 & Man-Whitney & $<.01$ \\
$\begin{array}{l}\text { Échelle violence physique } \\
\text { mineure (médiane) } \\
\begin{array}{l}\text { Échelle violence physique } \\
\text { sévère (médiane) }\end{array}\end{array}$ & 0 & 0 & Man-Whitney & $>.05$ \\
\hline $\begin{array}{l}\text { Score total au CTS } \\
\text { (moyenne) }\end{array}$ & 26.14 & 23.21 & Test-t & $>.05$ \\
\hline
\end{tabular}

\section{Discussion}

Les résultats formels obtenus à l'aide du DES et du Hand Test infirment l'hypothèse d'un plus grand nombre d'indices dissociatifs pour le groupe $\mathrm{H}$ comparativement au groupe $\mathrm{NH}$. Par contre, certaines données descriptives de l'incident homicide soulèvent des questions qui demeurent sans réponse. Ainsi, au moment de l'homicide, le contexte de perte de l'objet est présent dans la majorité des cas $(85,7 \%)$, les participants homicides mentionnant des symptômes rattachés à la dissociation dans $57 \%$ des cas lors de l'entrevue.

L'écart retrouvé entre les mesures formelles des indices dissociatifs (DES et Hand Test), d'une part, et les résultats descriptifs mentionnés cidessus, d'autre part, ne peut être expliqué par la faiblesse psychométrique des instruments de mesure. Le DES obtient de bons coefficients de fidélité et de cohérence interne ; au Hand Test, l'accord interjuges est bon.

Cet écart ne pourrait-il pas être expliqué par une restructuration psychique retrouvée chez les individus homicides? Celle-ci serait déclenchée par l'impact psychologique de l'acte homicide. En effet, on peut supposer que, durant les années qui ont suivi l'homicide, ces hommes ont entamé un travail de réorganisation psychologique afin d'intégrer dans leur psychisme l'acte homicide. Cette hypothèse prévaut dans un cadre théorique d'orientation dynamique. Cormier et al. (1971) précisent que, à la différence du psychotique, le non-psychotique, ce qui 
est le cas des «borderlines », revient à la réalité presque immédiatement après l'homicide. Il est alors confronté à la réalité qu'il a tué ; une donnée qu'il doit intégrer à la représentation qu'il a de lui-même, représentation qui s'accompagne d'un affect essentiellement dépressif. Ainsi, la problématique de la dissociation ne reposerait pas sur des indices aussi stables que nous l'avions pensé au départ. Les participants du groupe homicide n'ont pas été rencontrés immédiatement après le crime ; pour une majorité, l'événement datait déjà de plusieurs années $(M=5$ ans). De plus, la majorité des participants homicides $(69 \%)$ ont bénéficié d'une aide psychologique durant plus de 12 mois suite à l'homicide, ce qui est significativement plus élevé que pour le groupe témoin. Par contre, il est impossible de préciser la qualité de cette aide ; la forme d'aide est aussi très variable. L'impact de l'aide psychologique sur la dynamique interne des participants est questionnable ; la fréquence des rencontres est généralement faible (rarement plus d'une fois par semaine), ce qui est généralement considéré comme une intervention de soutien. En somme, ce ne serait pas tant l'aide psychologique que le délai écoulé entre le crime et la prise des mesures qui serait l'élément important. Ce délai a pu atténuer les indices dissociatifs, si l'on admet qu'ils étaient présents au moment du délit.

L'écart retrouvé entre les groupes sur le score de violence physique mineure au CTS est un résultat intéressant. Les participants $\mathrm{NH}$ ont un score significativement plus élevé de violence physique mineure au CTS que le groupe $\mathrm{H}$. On peut faire l'hypothèse que les individus $\mathrm{NH}$, parce qu'ils expriment plus fréquemment leur agressivité par des actes de violence physique mineure, sont moins à risque d'exploser à travers des actes de violence sévère tels que l'homicide de la conjointe. Inversement, les individus $\mathrm{H}$ ont peut-être plus tendance à accumuler leurs sentiments agressifs, possiblement pour éviter la rupture conjugale ; lorsqu'ils sont confrontés à la séparation, ils déchargent sur leur partenaire tous les sentiments d'agressivité accumulés pendant la relation. Toutefois, il importe de tenir compte du fait que les informations recueillies auprès des participants $\mathrm{H}$ portent sur des événements qui se situent plus loin dans le temps que les participants $\mathrm{NH}$; il est possible que des difficultés liées au souvenir interviennent. Il s'agit d'une limite de l'étude. Néanmoins, cette hypothèse est envisageable en regard des résultats observés par Gauthier (2000), lequel a constaté que les participants «borderlines » qui ont été violents physiquement envers leur conjointe démontrent moins de souffrance psychologique (indices Rors- 
chach) que les participants «borderlines " non violents ; le passage à l'acte violent favoriserait une décharge des tensions internes.

En outre, la consommation de substances psychoactives lors des incidents violents est présente dans des proportions considérables, constatation qui correspond aux études antérieures. Toutefois, il est difficile d'expliquer le rôle exact que jouent l'alcool et la drogue lors des incidents de violence mortelle et de violence non mortelle. Les substances psychoactives peuvent favoriser la perte d'inhibition face à la violence et pourraient aussi influencer le passage vers un état dissociatif. Par contre, ce n'est évidemment pas un facteur essentiel pour en arriver à l'homicide, puisque $50 \%$ des participants homicides n'avaient pas consommé ces substances au moment du crime.

Par ailleurs, l'étude comporte des limites. Premièrement, le petit nombre de participants étudiés $(N=28)$ ne permet pas d'atteindre une puissance statistique vraiment satisfaisante. Deuxièmement, le taux de refus est élevé, particulièrement pour le groupe $\mathrm{NH}$. Ce taux s'explique par un nombre élevé d'abandons suite à la première rencontre avec les participants. Nous pensons que le contenu de la première entrevue a pu être dérangeant sur le plan émotionnel pour certains. Ceci pourrait être relié au taux d'abandon retrouvé dans les thérapies pour hommes violents. Cette variable n'a par contre pas été mesurée dans l'étude.

Même si l'hypothèse de base n'est pas vérifiée, cette étude fournit des indices pour poursuivre une réflexion sur la violence conjugale. Premièrement, elle a le mérite de comparer deux formes de violence conjugale distinctes (mortelle et non mortelle), ce qui n'avait été fait que très rarement dans le passé, puisqu'on a tendance à étudier toutes les formes de violence conjugale sans les distinguer. Deuxièmement, elle permet de constater que le problème de la dissociation n'est pas aussi structural que nous l'avions pensé au départ. Dès lors, il importe que les études à venir évaluent les tendances dissociatives à un moment qui soit le plus rapproché possible de l'incident.

\section{Références}

BARnARD, G. W., VerA, H., VERA, M. et NEWMAN, G. 1982. "Till Death Do Us Part : A Study of Spouse Murder "Bulletin of the American Academy of Psychiatry and the Law 8 : 27 1-280.

BerGeret, J. 1995. Freud, la violence et la dépression, Paris : P.U.F. 
BernsteIN, E.M. et PUTNAM, M.D. 1986. "Development, Reliability and Validity of a Dissociation Scale " Journal of Nervous and Mental Disease $174: 727-735$.

BLINDER, M. 1984. “The Domestic Homicide » Family Therapy 11 : 185-198.

Blinder, M. 1985a. “The Domestic Homicide (part 2) » Family Therapy 12 : $1-24$

Blinder, M. 1985b. Lovers, Killers, Husbands and Wives, New York: StMartin's Press.

BLOCK, C. R. 1987. Lethal Violence at Home, Communication présentée au congrès annuel de l'American Society of Criminology, novembre, Montréal, Canada.

BOISVERT, R. et CUSSON, M. 1999. "Homicides et autres violences conjugales ", Pp. 77-90 in Les violences criminelles, sous la direction de J. Proulx, M. Cusson et M. Ouimet. Ste-Foy : Les presses de l'Université Laval, les Éditions de l'IQRC.

BARDEN, N. SCID traduction française, Sainte-Foy: Laboratoire de psychogénétique moléculaire, Centre Hospitalier de l'Université Laval.

BOUDOURIS, J. 1971 . "Homicide and the Family " Journal of Marriage and the Family 33 : 667-676.

Campbell, J. C. 1995. Assessing Dangerousness Violence by Sexual Offenders, Batterers, and Child Abusers, Thousand Oaks, Londres, New Delhi : Sage Publications.

CAMPBell, J. C. et LANDENBURGER, K. 1995. "Violence against Women ", Pp. 407-425 in Women's Health Care. A Comprehensive Handbook, sous la direction de C. I. Fogel et N. F. Woods. Thousand Oaks, Londres, New Delhi : Sage Publications.

Carlson, E. B. et Putnam, F. W. 1993. " An Update on the Dissociative Experiences Scale » Dissociation $6:$ 6-27.

Chimbos, P.D. 1978. Marital Violence : A Study of Interspouse Homicide, San Francisco: R \& E Associates.

Coleman, K. H., Weinman, M. L. et Bartholomew, P. 1980. "Factors Affecting Conjugal Violence " The Journal of Psychology 105 : 197-202.

COOPER, M. et EAVES, D. 1996. « Suicide Following Homicide in Family » Violence and Victims $11: 99-112$.

CORMIER, B. M. 1962. "Psychodynamics of Homicide Committed in a Marita! Relationship " Corrective Psychiatry and Journal of Social Therapy 8 (1) : 187-194.

CORMIER, B. M., AUGLIKER, C. C. J., BOYER, R., KENNEDY, M. et MERSEREAU, G. 1971. "The Psychodynamics of Homicide Committed in a Specific Relationship " Canadian Journal of Criminology 13 (1) : 1-8.

CôTÉ, A. 1991. La rage au cơur, rapport de recherche sur le traitement judiciaire de l'bomicide conjugal au Québec, Baie Comeau : Regroupement des femmes de la Côte Nord. 
CÔTÉ, G. et HODGiNS, S. 1992. “ The Prevalence of Major Mental Disorders among Homicide Offenders "International Journal of Law and Psychiatry $15: 89-99$.

CôtÉ, G., HOdGINS, S. et TOUPIN, J. 1999. "Psychopathie, comportement antisocial et violence " in Les violences criminelles, sous la direction de J. Proulx, M. Cusson et M. Ouimet. Ste-Foy : Les presses de l'Université Laval, les Éditions de l'IQRC.

CRAWFORD, M. et GARTNER, R. 1992. Woman Killing : Intimate Femicide in Ontario, 1974-1990, Toronto, Ontario: Women's directorate, Ministry of social services.

Daly, M. et WILSON, M. 1988. Homicide, New York : Aldine de Gruyter.

DANIEL, A. E. et HOLCOMB, W. R. 1985. « A Comparison between Men Charged with Domestic and Nondomestic Homicide " Bulletin of the American Academy of Psychiatry and the Law $13: 233-241$.

Dubester, K. A. et Braun, B. G. 1995. "Psychometric Properties of the Dissociative Experiences Scale " Journal of Nervous and Mental Disedse 183 : 231-235.

Duncan, J. W. et DunCan, G. M. 1978. "Murder in the Family », Pp. 171186 in Violence: Perspectives on Murder and Aggression, sous la direction de I. L. Kutash, S. B. Kutash et L. B. Schlesinger and Associates. San Francisco : Jossey-Bass.

DuTTON, D. G. 1998. The Abusive Personality Violence and Control in Intimate Relationships, New York, Londres : Guilford.

Dutton, D. G. et Golant, S. K. 1996. De la violence dans le couple, Paris : Bayard Éditions.

DUTTON, D. G., STARZOMSKI, A. et RYAN, L. 1996. "Antecedents of Abusive Personality and Abusive Behavior in Wife Assaulters " Journal of Family Violence $11:$ 113-132.

DUTTON, D. G., VAN GINKEL, C. et LANDOLT, M. A. 1996. " Jealousy, Intimate Abusiveness, and Intrusiveness " Journal of Family Violence 11 : 411-423.

EdLESON, J. L., EISIKOVITS, Z. et GUTTMANN, E. 1985. «Men Who Bater Women : A Critical Review of the Evidence " Journal of Family Issue 6 : 229-247.

EWING, C. P. 1997. Fatal Families: The Dynamics of Intrafamilial Homicide, Thousand Oaks, London, New Delhi : Sage Publications.

FIRST, M. B., GIBBON, M., SPITZER, R. L. et WILliAMS, J. B. W. 1997. Structured Clinical Interview for DSM-IV Axis I disorders : Clinical version, Washington : American Psychiatric Press Inc.

FirST, M. B., GIBBON, M., SPITZER, R. L., WILliaMS, J. B. W. et BENJAMIN, L. 1997. Structured Clinical Interview for DSM-IV Axis II disorders : Clinical version, Washington : American Psychiatric Press, Inc.

GAuThier, A. 2000. Comparaison des sujets limites ayant présenté des conduites agressives envers leur conjointe avec ceux n'ayant pas manifesté ce type de comportements (indices Rorschach), Université du Québec à Trois-Rivières : mémoire de maîtrise non publié. 
GeLLes, R. J. 1980. "Violence in the Family: A Review of Research in the Seventies " Journal of Marriage and the Family $42: 873-885$.

Gleaves, D. H., EBerenZ, K. P., WARNER, M. S. et FINE, C. G. 1995. "Measuring Clinical and Non-clinical Dissociation: A Comparison of the DES and QED " Dissociation $8: 24-31$.

Goetting, A. 1989a. « Men Who Kill their Mates : A Profile " Journal of Family Violence 4 : 285-296.

Goetting, A. 1989b. "Patterns of Marital Homicide: A Comparison of Husbands and Wives » Journal of Comparative Family Studies 20 :341-354.

GONDOLF, E. W. et SHESTAKOV, D. 1997. «Spousal Homicide in Russia versus the United States : Preleminary Findings and Implications " Journal of Family Violence $12: 63-74$

Gunderson, J. G. 1984. Borderline Personality Disorder, Washington : American Psychiatric Press, Inc.

Hamberger, L. K. et Hasting, J. E. 1986. "Personality Correlates of Men Who Abuse their Partners : A Cross-validations Study " Journal of Family Violence 1 : 323-341.

HAMBERGER, L.K. et HASTING, J. 1988. «Characteristics of Male Spouse Abusers Consistent with Personality Disorders " Hospital and Community Psychiatry 39 : 763-770.

Holzworth-munroe, A. et STUART, G. L. 1994. "Typologies of Male Batterers : Three Subtypes and the Differences among Them " Psychological Bulletin 116 : 476-497.

HoroszowsKI, P. 1973. " Homicide of Passion and its Motives ", Pp. 3-23 in Victimology : A new focus, Violence and victims (vol. 4), sous la direction de I. Drapkin et E. Viano. Lexington : Dc Heath and Co.

Hotaling, G. T. et SUGarman, D. B. 1986. «An Analysis of Risk Markers in Husband to Wife Violence : The Current State of Knowledge »Violence and Victims 1 : 101-124.

Kalichman, S. C. 1988. "MMPI Profiles of Women and Men Convicted of Domestic Homicide " Journal of Clinical Psychology 44 : 847-853.

KERNBERG, O. F. 1976. Borderline Conditions and Pathological Narcissism, NewYork : Aronson.

KRATCOSKI, P. C. 1987. « Families Who Kill » Marriage and Family Review 12 $(1-2): 47-70$.

LAFERrIÈRE, S. et BOUCHARD, C. 1993. "Validité discriminante du Questionnaire sur la résolution des conflits (QRC) : une étude comparative entre adultes en situation de violence et de non-violence " Les cabiers de recherche 2(3), Montréal : Université du Québec à Montréal, LAREHS.

LESTER, D. 1975. Crime of Passion : Murder and the Murderer, Chicago : NelsonHall.

Malarewitz, J. A. 1990. "Multiple Personality in French-speaking Countries", Pp. 32 in Proceedings of the Seventh International Conference on 
Multiple Personality and Dissociative State, sous la direction de B. G. Braun et E. B. Carlson. Chicago : Rush.

MaLmQUiST, C. P. 1980. "Psychiatric Aspects of Familicide " Bulletin of the American Academy of Psychiatry and the Law 8 : 298-304.

OKEEFE, M. 1997. «Incarcerated Battered Women : A Comparison of Battered Women Who Killed their Abusers and those Incarcerated for other Offenses " Journal of Family Violence 12 : 1-19.

POLK, K. 1994. "Scenarios of Masculine Violence in the Context of Sexual Intimacy ", Pp. 27-57 in When men kill, sous la direction de K. Polk. Cambridge : Cambridge University Press.

RosenberG, M. L. et MERCY, J. A. 1986. « Homicide : Epidemiologic Analysis at the National Level "Bulletin of New York Academy of Medecine 62 : 376-399.

Rosenbaum, M. 1990. "The Role of Depression in Couples Involved in Murder-suicide and Homicide " American Journal of Psychiatry 147 : 1036 1039.

Ross, C. A., Ellason, I. W. et ANDERSON, G. 1995. " A Factor Analysis of the Dissociative Experiences Scale (DES) in Dissociative Identity Disorder " Dissociation $8: 229-235$.

SAUNDERS, D. G. 1992. «A Typology of Men Who Batter : Three Types Derived from Cluster Analysis » American Journal of Orthopsychiatry 62 : 264275.

Saunders, D. G. 1995. "Prediction of Wife Assault ", Pp. 68-95 in Assessing Dangerousness Violence by Sexual Offenders, Batterers, and Child Abusers, (vol. 8), sous la direction de J. C. Campbell. Thousand Oaks, Londres, New Delhi : Sage Publications.

Saunders, D. G. et Browne, A. 1991. "Domestic Homicide », Pp. 379-402 in Case Studies in Family Violence, sous la direction de R.T. Ammerman et M. Hersen. New York, Londres : Plenum Press.

SHOWALTER, C. R., BONNIS, R. J. et RODDY, v. 1980. « The Spousal-homicide Syndrome $»$ International Journal of Law and Psychiatry 3 : 117-141.

SCHUMM, W. R. S. et BAGAROzZI, D. A. 1989. "Family Measurement Techniques » The American Journal of Family Therapy $17: 165-168$.

STATISTIQUes CANADA. 1994. "Résultats d'une enquête nationale sur l'agression contre la conjointe " Juristat 14 (9).

STEINMETZ, S. K. 1978. "Wife Beating : A Critical and Reformulation of Existing Theory " Bulletin of American Academy of Psychiatry and the Law 6 : 322-334.

STRAUS, M. A. 1979. « Measuring Intrafamily Conflict and Violence: The Conflict Tactics (CT) Scales " Journal of Marriage and The Family $41: 75$ 88.

Straus, M. A. 1986. "Domestic Violence and Homicide Antecedents » Bulletin of the New York Academy of Medicine 62 : 446-465. 
Straus, M. A. et Gelles, R. J. 1990. Physical Violence in American Families. Risk Factors and Adaptations to Violence in 8,145 Families, Nouveau-Brunswick : Transaction Publishers.

Sugarman, D. B., ALDARONDO, E. et BONEY-MCCOY, s. 1996. " Risk Marker Analysis of Husband-to-wife Violence : A Continuum of Aggression " Journal of Social Applied Psychology 26 : 313-337.

TOLMAN, R. M. et BENNETT, L. W. 1990. « A Review of Quantitative Research on Men Who Batter » Journal of Interpersonal Violence 5 : 87-118.

VAN IJZENDOORN, M. H. et SCHUENGEL, C. 1996. "The Measurement of Dissociation in Normal and Clinical Populations : Meta-analytic Validation of the Dissociative Experiences Scale (DES) " Clinical Psychology Review $16: 365-382$.

Walker, L. E. et MeloY, J. R. 1998. "Stalking and Domestic Violence», Pp. 139-161 in The Psychology of Stalking Clinical and Forensic Perspectives, sous la direction de G. R. Meloy. San Diego : Academic Press.

WaGner, E. E. 1983. The Hand Test Manual Revised 1983 (éd. rev.), Los Angeles : Western Psychological Services.

WILSON, M. et DALY, M. 1993. "Spousal Homicide Risk and Estrangement " Violence and Victims $8: 3-16$.

WILSON, M., JOHNSON, H. et DALY, M. 1995. “ Lethal and Nonlethal Violence against Wives " Canadian Journal of Criminology $37:$ 331-361.

Wolfgang, M. E. 1958. Patterns in Criminal Homicide, Philadelphia : University of Pennsylvania Press.

YARVIS, R. M. 1990. "Axis I and Axis II Diagnostic Parameters of homicide "Bulletin of American Academy Psychiatry and the Law 18 : 249 269.

\section{Note des auteurs}

Les auteurs expriment leur gratitude à Mesdames Suzanne Léveillé et Annie Gauthier pour leur collaboration. Ils adressent également leurs remerciements aux membres du personnel du Service correctionnel du Canada région Québec, du centre le CAHO et du centre Accord Mauricie Inc. qui ont permis la sélection de participants. Ils tiennent aussi à exprimer leur reconnaissance au Centre le Parcours pour le prêt de ses locaux, et, bien sûr, à tous les participants pour ieur générosité. 